\title{
Return of norovirus and rotavirus activities in winter 2020/21 in an Asian city with strict zero COVID-19 control strategy, Hong Kong, China
}

Martin Chi-Wai Chan ( $\square$ martin.chan@link.cuhk.edu.hk)

https://orcid.org/0000-0002-1568-1596

\section{Short Report}

Keywords: COVID-19, norovirus, rotavirus, seasonality, viral gastroenteritis

Posted Date: November 3rd, 2021

DOI: https://doi.org/10.21203/rs.3.rs-1045089/v1

License: (c) (i) This work is licensed under a Creative Commons Attribution 4.0 International License.

Read Full License

Version of Record: A version of this preprint was published at Emerging Infectious Diseases on January 19th, 2022. See the published version at https://doi.org/10.3201/eid2803.212117. 


\section{Abstract}

We observed active norovirus and rotavirus circulation in winter 2020/21 in Hong Kong while a variety of non-pharmaceutical interventions to eliminate COVID-19 were effective. The initial collateral benefit of countermeasures for COVID-19 to reduce viral gastroenteritis burden is not sustainable even in a city with continual zero COVID-19 control strategy.

\section{Introduction}

The unfolding novel coronavirus disease 2019 (COVID-19) pandemic is an unprecedented public health crisis in the modern history of humankind. One collateral consequence of the COVID-19 pandemic is concomitant rapid decline in the incidence of viral gastroenteritis in the first year of the COVID-19 pandemic, as observed in multiple countries such as China (1), US (2), England (3), Germany (4), Japan (5) and Australia (6). The most likely explanations were sliced testing capacity that led to underreporting and wide implementation of non-specific non-pharmaceutical interventions for COVID-19 such as frequent handwashing and physical distancing that reduced human-to-human transmission of virus. Hong Kong is a metropolitan city in South China and has been continuously implementing stringent and effective elimination (also known as zero COVID) strategy to suppress the importation and local spread of COVID-19 since the start of the pandemic. Importantly, the local routine laboratory syndromic surveillance on viral gastroenteritis remained largely unaffected in the pandemic and testing capacity for common diarrheagenic viruses was only mildly reduced, providing a well-controlled setting to study the epidemiology of viral gastroenteritis in the COVID-19 era. Here, we reported that the otherwise typical winter 2019/20 season of norovirus and rotavirus ended abruptly and earlier than usual in Hong Kong soon after the initial global spread of COVID-19, but both viruses returned in winter 2020/21 amid the local continual strict zero COVID-19 control strategy.

\section{The Study}

Local territory-wide monthly laboratory data on the PCR-based detection of norovirus and rotavirus, the two leading causes of viral gastroenteritis (7), are publicly available since January 2013 from the Centre for Health Protection of Hong Kong (equivalent in function to other national public health agencies such

as the China $\mathrm{CDC}$ ) (8). Laboratory data for less common diarrheagenic viruses including sapovirus, astrovirus and enteric adenovirus were available from May 2017 onwards. From January 2013 through September 2021, a total of 104,187 stool specimens collected from sporadic and outbreak cases of acute gastroenteritis were tested. The median number of specimens tested each month was 1,008 (interquartile range [IQR]: 912-1,114) and 872 (IQR: 784-990) before and during the COVID-19 pandemic, respectively. Although an average of $13.5 \%$ less stool specimens were tested during the pandemic $(P<0.01$; MannWhitney $U$ test), the reduced sample sizes were still of sufficient power to detect at least one positive specimen under a virus prevalence as low as $0.5 \%$ (namely 1 in 200) at a $95 \%$ confidence level. 
Monthly positive rate of the five common viral causes of acute gastroenteritis is shown in the Figure. During winter $2019 / 20$, the positive rate of rotavirus dropped abruptly from peak by $70 \%$ in February 2020 , shortly after the initial global spread of COVID-19 and remained at a much lower level of $0.1 \%-$ $0.6 \%$ through September 2020 compared with a median of 5.4\% (IQR: $2.8 \%-13.4 \%$ ) during the same period in the past seven years. The winter $2019 / 20$ season of rotavirus ended about two months earlier than usual. Considering that the observed lower positive rate may be confounded by the declining trend in recent years with the availability of rotavirus vaccines, we further examined data on norovirus in which no effective antivirals or vaccines are presently available. Likewise, norovirus positive rate dropped sharply from peak by $56 \%$ in February 2020 and remained at a much lower level of $0.3 \%-1.5 \%$ through September 2020 compared with a median of $6.4 \%$ (IQR: $5.2 \%-10.0 \%$ ) during the same period in the past seven years. The winter 2019/20 season of norovirus ended almost three months earlier than usual. We have previously shown that norovirus exhibited a bimodal seasonality in Hong Kong with two peaks every year, one in winter and another in summer/autumn (9). Of note, the expected norovirus peak in summer/autumn 2020 was not observed in this study. For sapovirus, astrovirus and enteric adenovirus, these viruses became hardly detectable since the start of the COVID-19 pandemic, showing a positive rate of persistently below $1 \%$ throughout 2020 and 2021.

In winter 2020/21, a typical seasonal peak of norovirus with a positive rate of $10.3 \%$ was observed in January 2021, and the rate was comparable to the median of $14.4 \%$ in the past seven winter seasons. Likewise, a typical seasonal peak of rotavirus with a positive rate of $4.8 \%$ was observed in January 2021 , and the rate was highly comparable to that of $5.3 \%$ and $6.2 \%$ in the past two winter seasons, albeit on a progressively decreasing trend in recent years. These data indicated active circulation of norovirus and rotavirus in the community during winter 2020/21 while strict non-pharmaceutical interventions for COVID-19, including work-from-home arrangement for civil servants, mandatory mask-wearing outside homes, school closure and prohibition on group gatherings of more than four persons in public places, were in effect in the city.

\section{Conclusions}

We observed an abrupt decline in activities of multiple diarrheagenic viruses, in particular norovirus and rotavirus, and shortening of their seasons soon after the initial global spread of COVID-19 in early 2020. Hong Kong has adopted a multi-pronged elimination strategy to contain COVID-19 since the local report of the first case in January 2020 (10) and maintained one of the world's lowest infection rates so far ( $<0.2 \%$ of the local population). Considering viral gastroenteritis was primarily transmitted through person-to-person contact, non-pharmaceutical interventions for COVID-19 such as social distancing might have inadvertently stopped the spread of non-respiratory pathogens. The possibility of underreporting was excluded due to the uninterrupted laboratory syndromic surveillance on viral gastroenteritis in Hong Kong. While return of viral gastroenteritis is anticipated in countries implementing mitigation strategy accompanied with relaxation of infection control measures, the seasonal activities of norovirus and rotavirus observed in winter 2020/21 in Hong Kong was to some extent unexpected. This is unlikely explained by pandemic fatigue as the local seasonal influenza activity remained at an unprecedented 
virtually zero level in winter 2020/21 (11). Other factors such as waning immunity and thus accumulation of susceptible population may come to play. This study is limited by the lack of virus characterization to determine whether the rise of viral gastroenteritis was a result of emergence of new strains, especially for norovirus in which new immune-escaped strains emerged periodically (12). Furthermore, additional analysis on the route of transmission of cases would be helpful as public health interventions for COVID19 may be less effective to diarrheagenic viruses that can spread via food-/water-borne routes.

In conclusion, the initial collateral benefit of non-pharmaceutical interventions for COVID-19 to reduce the burden of viral gastroenteritis is not sustainable even in a city with continual zero COVID-19 control strategy.

\section{References}

1. Wang LP, Han JY, Zhou SX, Yu LJ, Lu QB, Zhang XA, et al. The changing pattern of enteric pathogen infections in China during the COVID-19 pandemic: a nation-wide observational study. Lancet Reg Health West Pac. 2021 Nov; 16:100268.

2. Kraay ANM, Han P, Kambhampati AK, Wikswo ME, Mirza SA, Lopman BA. Impact of nonpharmaceutical interventions for severe acute respiratory syndrome coronavirus 2 on norovirus outbreaks: an analysis of outbreaks reported by 9 US States. J Infect Dis. 2021 Jul 2;224(1):9-13.

3. Ondrikova N, Clough HE, Douglas A, Iturriza-Gomara M, Larkin L, Vivancos R, et al. Differential impact of the COVID-19 pandemic on laboratory reporting of norovirus and Campylobacter in England: $A$ modelling approach. PLoS One. 2021;16(8):e0256638.

4. Eigner U, Verstraeten T, Weil J. Decrease in norovirus infections in Germany following COVID-19 containment measures. J Infect. 2021 Jun;82(6):276-316.

5. Fukuda Y, Tsugawa T, Nagaoka Y, Ishii A, Nawa T, Togashi A, et al. Surveillance in hospitalized children with infectious diseases in Japan: Pre- and post-coronavirus disease 2019. J Infect Chemother. 2021 Nov;27(11):1639-47.

6. Bruggink LD, Garcia-Clapes A, Tran T, Druce JD, Thorley BR. Decreased incidence of enterovirus and norovirus infections during the COVID-19 pandemic, Victoria, Australia, 2020. Commun Dis Intell. 2021 Jan 29;45.

7. Banyai K, Estes MK, Martella V, Parashar UD. Viral gastroenteritis. Lancet. 2018 Jul 14;392(10142):175-86.

8. Detection of gastroenteritis viruses from faecal specimens. [cited 2021-10-11]; Available from: https://www.chp.gov.hk/en/statistics/data/10/641/717/3957.html

9. Chan MC, Kwok K, Zhang LY, Mohammad KN, Lee N, Lui GCY, et al. Bimodal seasonality and alternating predominance of norovirus GII.4 and non-GII.4, Hong Kong, China, 2014-2017. Emerg Infect Dis. 2018 Apr 17;24(4).

10. Lam HY, Lam TS, Wong CH, Lam WH, Leung CME, Au KWA, et al. The epidemiology of COVID-19 cases and the successful containment strategy in Hong Kong-January to May 2020. Int J Infect Dis. 
2020 Sep;98:51-8.

11. Detection of pathogens from respiratory specimens. [cited 2021-10-12]; Available from: https://www.chp.gov.hk/en/statistics/data/10/641/642/2274.html

12. Parra GI. Emergence of norovirus strains: A tale of two genes. Virus Evolultion. 2019 Jul;5(2):vez048.

\section{Declarations}

CONFLICT OF INTEREST

None declared.

\section{ACKNOWLEDGMENTS}

The work received no internal or external funding support.

\section{ABOUT THE AUTHOR}

Dr. Chan was a senior scientific reviewer at the Research Office of the Food and Health Bureau of Hong Kong and an assistant professor in the Department of Microbiology and Stanley Ho Centre for Emerging Infectious Diseases of the Chinese University of Hong Kong. His research interests are molecular epidemiology and pathogenesis of intentinal and respiratory viral infections, especially those caused by noroviruses and influenza viruses.

\section{Figures}




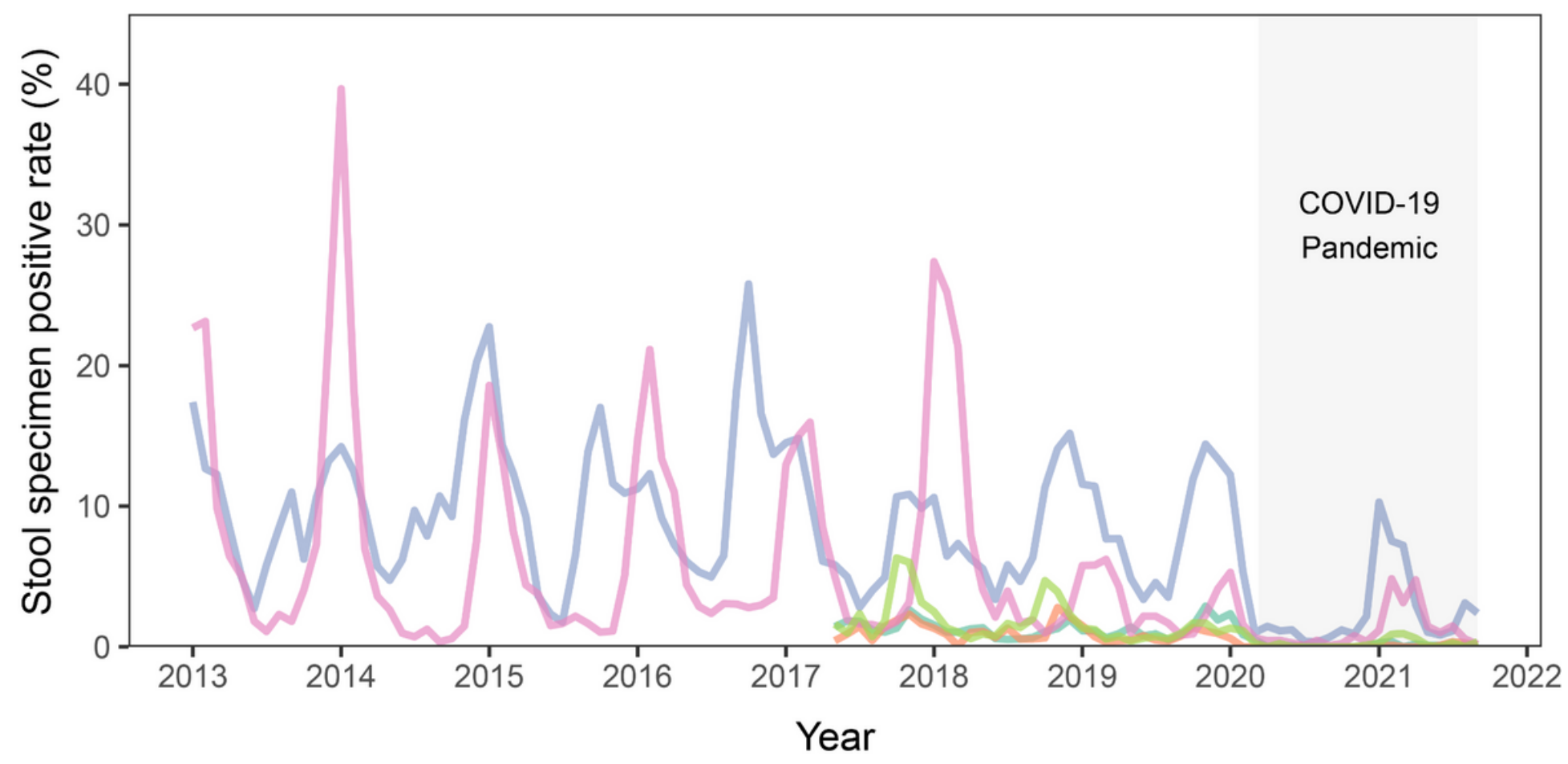

- Astrovirus - Enteric Adenovirus - Norovirus - Rotavirus - Sapovirus

\section{Figure 1}

Monthly positive rate of five common viral causes of acute gastroenteritis in stool specimens from routine, territory-wide, PCR-based laboratory syndromic surveillance data, January 2013-September 2021, Hong Kong, China. Data on sapovirus, astrovirus and enteric adenovirus were available from May 2017 onwards. The first coronavirus disease 2019 (COVID-19) case in Hong Kong was reported on 23 January 2020 and COVID-19 was declared pandemic by the World Health Organization on 11 March 2020. 